EPSC Abstracts

Vol. 14, EPSC2020-644, 2020

https://doi.org/10.5194/epsc2020-644

Europlanet Science Congress 2020

(C) Author(s) 2021. This work is distributed under

the Creative Commons Attribution 4.0 License.

\title{
SSHADE, its solid spectra databases and its future band list of molecular solids
}

Bernard Schmitt ${ }^{1}$, Philippe Bollard ${ }^{1}$, Damien Albert ${ }^{2}$, Lydie Bonal ${ }^{1}$, Olivier Poch ${ }^{1}$, and the SSHADE Consortium*

${ }^{1}$ CNRS / Univ. Grenoble Alpes, Institut de Planétologie et Astrophysique de Grenoble, Grenoble Cedex 9, France

(bernard.schmitt@univ-grenoble-alpes.fr)

${ }^{2}$ CNRS / Univ. Grenoble Alpes, Observatoire des Sciences de I'Univers de Grenoble, Grenoble Cedex 9, France

${ }^{*}$ A full list of authors appears at the end of the abstract

The SSHADE database infrastructure (http://www.sshade.eu) hosts spectral data of many different types of solids: ices, snows, minerals, carbonaceous matters, meteorites, IDPs and other cosmomaterials, $\ldots$ covering a wide range of wavelengths: from $X$-rays to millimeter wavelengths.

Its Search / Visualization / Export interface is open to the community since February 2018. It currently contains over 3500 spectra.

We are currently developping a 'band list' database that will provide the band parameters (position, width, intensity, isotopic species, attribution mode, ...) of a number of solids of astrophysical and planetary interest in various phases (crystalline, amorphous, ...) and mixtures (different compositions) and at different temperatures or pressures. We will first feed this database from critical compilations of all data published in various journals for pure ices and molecular solids (hydrates, clathrates, sulfur compounds, ...) and their mixture, including the own works of the members of the SSHADE consortium.

An efficient search tool will allow to filter on various parameters such as band position (or spectral range) and intensity, expected molecular or atomic formula, type of vibration (e.g. infrared, Raman, fluorescence...) and display the results graphically. A demonstrator prototype is online in the 'old GhoSST' database (https://ghosst.osug.fr/search/band).

This band list will be a key tool for astronomers and space explorers to identify unknown absorption bands observed in the spectra of the surface or atmosphere of many objects in the solar system. Once the best candidate solid selected by the user the tool will point to the best relevant spectral data present in the SSHADE databases.

SSHADE Consortium: IPAG/UGA-CNRS (F), IAS/UPS (F), AIU Observatory (D), IRAP/U. Toulouse $(F)$, LPG/U. Nantes (F), SSDPG/SRC-PAS (PL) CML/IGS-PAS (PL), WP/Unibe (CH), FAME/ESRF (F), PIIM/U. Aix-Marseille (F), DPS/OU (GB), IAPS/INAF Roma (I), LISA/UPEC (F), IEM/CSIC (E), 
LATMOS/IPSL (F), LGL-TPE/ENS-Lyon (F), Konkoly Astro. Inst./CSFK (HU), PRL (IN), U. Parthenope (I), Univ. Vienna (AU), NSRRC (TW). 